\title{
COUNTEREXAMPLES IN THE THEORY OF NONSELFADJOINT OPERATOR ALGEBRAS
}

\author{
W. R. WOGEN
}

In this note we announce the answers to several questions which involve nonselfadjoint operator algebras. Detailed proofs will appear elsewhere.

We use the following notation. $\mathscr{H}$ is a separable Hilbert space, $B(\not H)$ is the algebra of bounded linear operators on $\mathcal{H}$, and $B_{1}(K)$ is the ideal of trace class operators on $\mathcal{H}$. For $T \in B(\mathscr{H}),\{T\}^{\prime}$ is the commutant of $T$ and $\{T\}^{\prime \prime}$ is the double commutant of $T$.

$B(\not H)$ is the dual of $B_{1}(\not H)$ (see [2]) so that $B(\not H)$ has a weak * topology. $A(T)$ denotes the smallest weak ${ }^{*}$ closed algebra containing $T$ and $I$, while $\mathcal{W}(T)$ is the smallest weak operator closed algebra containing $T$ and $I$. Lat $T$ is the lattice of (closed) invariant subspaces of $T$, and Alg Lat $T=\{B \in$ $B(\not{H})$ : Lat $T \subset$ Lat $B\}$. It is elementary that $A(T) \subset \mathcal{W}(T) \subset\{T\}^{\prime \prime} \subset\{T\}^{\prime}$, that $\mathcal{W}(T) \subset \operatorname{Alg} \operatorname{Lat} T$, and that all of these sets except $A(T)$ are weakly closed algebras. Further, $T$ is said to be reflexive if $\mathcal{W}(T)=\operatorname{Alg} \operatorname{Lat} T$.

We will consider the following questions.

QUESTION 1. Does $\mathcal{W}(T)=\{T\}^{\prime} \cap \operatorname{Alg}$ Lat $T, \forall T \in B(\not)$ ?

QUESTION 2. Does $\mathcal{W}(T)=\{T\}^{\prime \prime} \cap \operatorname{Alg} \operatorname{Lat} T, \forall T \in B(\not{H})$ ?

QUESTION 3. Must $T^{(n)}$ be reflexive, $\forall T \in B(\mathfrak{K})$ and $\forall n>1$ ? (Here $T^{(n)}$ denotes the direct sum of $n$ copies of $T$.)

QUESTION 4. If $T_{1}$ and $T_{2}$ are reflexive operators, must $T_{1} \oplus T_{2}$ be reflexive?

QUESTION 5. Does $A(T)=\mathcal{W}(T), \forall T \in B(\not)$ ?

QUESTION 6. Does $\mathcal{W}(T)$ have a separating vector, $\forall T \subset B(\mathcal{H})$ ?

Before stating the last question, we need some additional notation. Since $\mathcal{W}(T)$ is weak * closed in $B(\not), \mathcal{W}(T)$ is a dual space, with predual $\mathcal{W}(T)_{*}=$ $B_{1}(\mathcal{H}) / \mathcal{W}(T)_{\perp}$. Here $\mathcal{W}(T)_{\perp}$ denotes the preannihilator of $\mathcal{W}(T)$. For each $n$, let $F_{n} \subset B_{1}(\not H)$ denote the set of operators of rank $\leq n$.

QUESTION 7. Is $F_{1} / \mathcal{W}(T)_{\perp}$ dense in $\mathcal{W}(T)_{*}, \forall T \subset B(\not)$ ?

Some remarks regarding these questions are in order. There are some relations among the questions. For $n=1,2$, or 6 , an affirmative answer to Question $n$ implies an affirmative answer to Question $n+1$.

Question 1 was raised independently by D. Sarason and P. Rosenthal (see [6, p. 195] and [7]). Rosenthal also asked Question 2 in [7]. In [4], J. Deddens listed several open questions, including Questions 3 and 4 , concerning reflexive operators.

Question 5 has been raised by many people. The question appears in [2]. In [8], D. Westwood gave an example of an operator $T$ so that $A(T)=\mathcal{W}(T)$ but so that the weak and weak * topologies are different on $A(T)$.

Received by the editors February 1, 1986.

1980 Mathematics Subject Classification (1985 Revision). Primary 47C05, 47D25; Secondary 47A15. 
Questions 6 and 7 were raised by D. Larson in a private communication. The motivation for the questions arose from the following. There has been intense research activity (see $[\mathbf{1}, \mathbf{2}$, and 3], e.g.) on operators $T$ such that every weak * continuous linear functional on $\mathcal{W}(T)$ is represented by a rank one operator. (Thus $T$ satisfies $\mathcal{W}(T)_{*}=F_{1} / \mathcal{W}(T)_{\perp}$.) There are operators $T$ which do not have this property (see [5 and 1]), but for these operators $T$, $F_{1} / \mathcal{W}(T)_{\perp}$ is dense in $\mathcal{W}(T)_{*}$.

We have been able to show that all seven of these questions have a negative answer. The key to the construction of the counterexamples is the following theorem.

THEOREM. Let $\sharp$ and $K$ be separable Hilbert spaces with $\operatorname{dim} K=\infty$. Let $S$ be a weakly closed subspace of $B(\mathcal{H})$. Then there is an operator $T \in$ $B(\mathcal{H} \oplus K \oplus \mathcal{K})$ of form

$$
T=\left(\begin{array}{ccc}
0 & P & 0 \\
0 & W & Q \\
0 & 0 & 0
\end{array}\right)
$$

so that $\mathcal{W}(T)$ splits as an independent direct sum: $\quad \mathcal{W}(T)=B(T) \dot{+} \tilde{S}$, where $\tilde{S}=\left\{A \in B(H \oplus K \oplus \mathscr{H}): A_{1,3} \in S\right.$ and $A_{i, j}=0$ if $\left.(i, j) \neq(1,3)\right\}$ and $\mathrm{B}(T)=\left\{A \in \mathcal{W}(T): A_{1,3}=0\right\}$.

We now indicate how this theorem settles Question 1 . Let $\not=\mathbf{C}^{2}$ and let $S$ be the set of trace zero operators on $\nVdash$. Then $S$ is a transitive subspace of $B(\not H)$. This means (see [1]) that $S x=\not \forall$ for all $x \in \not{H}, x \neq 0$. Construct $T$ as in the theorem, so that $\mathcal{W}(T)=B(T) \dot{+}$. Now every $A \in \widetilde{B(\not H})$ is nonzero only in its $(1,3)$ entry, so $A T=T A=0$ and $A \in\{T\}^{\prime}$. Also, using transitivity of $S$, it is easy to see that $A \in \operatorname{Alg}$ Lat $T$. $S$ is a proper subspace, so $\widetilde{B(H)}$ is not contained in $\mathcal{W}(T)$ and we have a counterexample. We note that this example was motivated in part by the excellent survey of some finite dimension results which appears in the beginning of the paper [1] of E. Azoff.

It is easy to check that choosing $S=B(\not H)$ in the theorem yields a counterexample to Questions 6 and 7. Some additional information on the structure of the subspace $B(T)$ is required in order to give examples settling the remaining questions.

We now outline the proof of the theorem. We identify $K$ with $\bigoplus_{1}^{\infty} \nVdash$. In the matrix for $T$ let $P$ be the isometry of $\mathcal{H}$ into $K$ with matrix $\left(\begin{array}{llll}I & 0 & 0 & \cdots\end{array}\right)$. Let $W$ be a backward operator weighted shift with weight sequence $\left(w_{n} I\right)$ to be specified later. Thus $W$ has matrix $\left(W_{i, j}\right)$ where $W_{n, n+1}=w_{n} I, n \geq 1$, and all other entries $=0$. Let $C$ be a countable weakly dense set in the unit ball of $S$. Let $\left(Q_{n}\right)$ be a sequence in $C$ so that each $C \in C$ appears infinitely often in $\left(Q_{n}\right)$. Since $Q$ is to be an operator from $\mathcal{K}$ to $\mathcal{H}$, we think of $Q$ as an operator matrix with one column. Let the $n$th entry of this column be $b_{n} Q_{n}$. Here we assume $b_{n} \neq 0 \forall n$ and that $\left(b_{n}\right) \in l^{2}$. This insures that $Q$ is bounded. 
If $n \geq 1$, then

$$
T^{n+1}=\left(\begin{array}{ccc}
0 & P W^{n} & P W^{n-1} Q \\
0 & W^{n+1} & W^{n} Q \\
0 & 0 & 0
\end{array}\right)
$$

Now $P W^{n-1} Q=\lambda_{n} Q_{n}$, where $\lambda_{n}=w_{1} w_{2} \cdots w_{n-1} b_{n}$. Consider the sequence $\left(\left(1 / \lambda_{n}\right) T^{n+1}\right)$. If the weights $w_{n}$ are chosen to go to zero sufficiently quickly, then all matrix entries of $\left(1 / \lambda_{n}\right) T^{n+1}$ except for the $(1,3)$ entry go to zero with $n$. It follows that $\tilde{S} \subset \mathcal{W}(T)$.

Part of this work was done while the author attended the NSF-CBMS conference on "Optimization in Operator Theory, in Analytic Function Theory, and in Electrical Engineering" at the University of Nebraska, Lincoln. Thanks are due to the University, to D. Larson and G. Woodward, who coordinated the conference, and to the NSF for conference support. The author wishes to thank J. Conway, D. Larson, and E. Nordgren for helpful discussions during the conference.

\section{REFERENCES}

1. E. Azoff, On finite rank operators and preannihilators, preprint.

2. H. Bèrkovici, C. Foias, and C. Pearcy, Invariant subspaces, dilation theory, and dual algebras, CBMS Regional Conf. Ser. in Math., no. 56, Amer. Math. Soc., Providence, R. I., 1985.

3. S. Brown, Some invariant subspaces for subnormal operators, Integral Equations and Operator Theory 1 (1978), 310-333.

4. J. Deddens, Reflexive operators, Indiana Univ. Math. J. 20 (1971), 887-889.

5. D. Hadwin and E. Nordgren, Subalgebras of reflexive algebras, J. Operator Theory 7 (1982), 3-23.

6. H. Radjavi and P. Rosenthal, Invariant subspaces, Springer-Verlag, New York, 1973.

7. P. Rosenthal, Problems in invariant subspaces and operator algebras, Colloq. Math. Soc. János Bolyai, vol. 5, North-Holland, Amsterdam, 1970.

8. D. Westwood, Weak operator and weak * topologies on singly generated algebras, J. Operator Theory (submitted).

Department of Mathematics, University of North Carolina, Chapel HILL, NORTH CAROLINA 27514 